\title{
Operational Experience with a High Speed Video Data Acquisition System in Fermilab E-687.
}

\author{
A. E. Baumbaugh and K. L. Knickerbocker \\ Fermi National Accelerator Laboratory \\ P.O. Box 500, Batavia, Illinois 60510 \\ B. Baumbaugh and R. Ruchti \\ The University of Notre Dame \\ Notre Dame, Indiana 46556
}

October 21,1987

*Presented at the IEEE 1987 Nuclear Science Symposium, San Francisco, California, October 21-23, 1987 


\title{
OPERATIONAL EXPERIENCE WITH A HIGH SPEED VIDEO DATA ACQUISITION SYSTEM IN FERMILAB EXPERIMENT E-687.
}

\author{
A. E. Baumbaugh, and K. L. Knickerbocker \\ Fermi National Accelerator Laboratory," \\ Batavia, Illinois \\ B. Baumbaugh and R. Ruchti \\ University of Notre Dame, ${ }^{* *}$ Notre Dame, Indiana
}

\begin{abstract}
Operation of a high speed, triggerable, Video Data Acquisition System (VDAS) including a hardware data Acquisition System actor and 16 megabyte First-In-First-Out buffer memory (FIFO) will be discussed. Active target imaging techniques for High Energy Physics are described and preliminary experimental data is reported. The hardware architecture for the imaging system and experiment will be discussed as well as other applications for the imaging oystem. Date rates for the compactor is over 30 megabytes/sec and the FIFO has been run at 100 megabytes/sec. The system can be operated at standard video rates or at any rate up to 30 million pixels/second.
\end{abstract}

\section{Introduction}

The development of high resolution scintillating glass active targets[1] has created the need for specialized active targets (1 ] has crision systems. The data produced by these targets is in the form of an image, which is the projection of the interaction vertex upon the face of the active target. Image data of this type presents several problems which must be addressed. Images contain very large volumes of data which must be stored and processed, this requires high speeds if significant numbers of images are to be recorded. To be useful for HEP the system must also be triggerable. The use of film for this type of target is only useful for testing, as film cameras can, at best, run only a few frames/second in triggered mode. With these requirements in mind VDAS was developed. The system is flexible enough to accommodate virtually any video format, standard or non-standard, and fast enough to be useful in HEP. VDAS is capable of digitizing an image at 30 million pixels per second and can store many images in its internal FIFO buffer which can run at 100 megabytes/second. The firat VDAS system has been inatalled in the Wide Band Photon Hall at Fermilab as part of experiment E-687 and will be taking dats soon.

\section{Fxperiment 5-687}

Experiment-687 is designed to study the photo production of charm and beauty states. This is accomplished by a large multi-particle spectrometer and elaborate tracking system. The experiment will use tagged photons interacting in a glass or plastic scintillating fiber target for the primary vertex. The active target will study short-lived decays and aid in identification of decay products.

* Operated by Universities Research Association Inc,. under contract with the United States Department of Energy.

* This work partially funded by The National Science Foundation.

\section{Spectrometer:}

A diagram of the spectrometer for E-687 is shown in Figure 1. The task of detecting particles emerging from primary interactions occurring in the target and secondary decays in and outside the target is accomplished through use of a high rate, multi-particle spectrometer. The E-687 spectrometer roughly divides its job into two regimes: an inner or forward portion for low angle tracks and an outer portion for high angle tracks. In both regimes electromagnetic calorimetry and muon detection systems are provided, with hadron calorimetry for the inner region only. The tracking system consists of twenty planes of proportional wire chambers together with a twelve plane silicon microstrip detector for additional tracking in the vertex region. The spectrometer employs two analyzing magnets (of opposite polarity) with vertical bend planes, three Cerenkov detectors for particle identification, beam calorimetry devices (not shown) as well as miscellaneous scintillation trigger counters and hodoscopes.

The spectrometer will provide the tracking and particle identification for all particles produced in the interaction, however, the tracking capability is not precise enough to detect particle decays which happen very close to the primary interaction vertex. For this reason the target used will be an active or instrumented one. The spectrometer can record data faster than the active target, so only a subset of the spectrometer dats will also have interaction vertex image data. The trigger will be set up so that the most interesting candidate events will gate the imaging system.

\section{Active Target:}

The active target syatem is shown in Figure 2. It consists of a scintillating fiber target, a multiple stage image intensifier, camera, the Video Data Acquiaition System and data recording system. There is also a crude image processing and display system for event viewing.

The active target is made up of approximately 1 million GS1 Cerium glass fibers fused into a cube twenty millimeters on side. Although this target is glase, plastic fibers could also be used to manufacture a target. (The development of this type of plastic fibers is under way. (2 I) A target constructed of these plastic fibers would have inherent advantages in a photon beam as plastic has a longer radiation length than glass.

The image produced by the target is a twodimensional projection of the interaction region. This image has extremely low light levels, essentially single photon counting. This requires the use of a multistage image intensifier/expander of the GEN-1 type, with a gain of approximately 100,000 to produce light levels usable for the camera. The image is expanded prior to intensification and reduced afterward to minimize the spot sixe effects of the image intensifiers.

The system also includes a fast local trigger[3] and gated micro-channel plate stage in the intensifier so that only selected events are allowed to be gated onto the photo-sensitive face of the imaging device. The time 
delay needed for trigger decioions is provided by the phosphor decay time in each of the intensifier stages. These phosphors are chosen to match the beam rates of the experiment and the projected trigger rates.

The first data taking run will use a Silicon Intensified Target, or SIT, Vidicon camera as the primary imaging device. The electroatatically deflected SIT has the advantage of being triggerable, the camera can start read-out cycle at any time, unlike a magnetically deflected camers in which the deflection coils are part of an oscillator. The electrostatic deflection allows the SIT to scan at a higher rate, as there is little energy stored in the deflection circuits. The SIT also has reasonably high resolution, effectively 768 by 512 pixels.
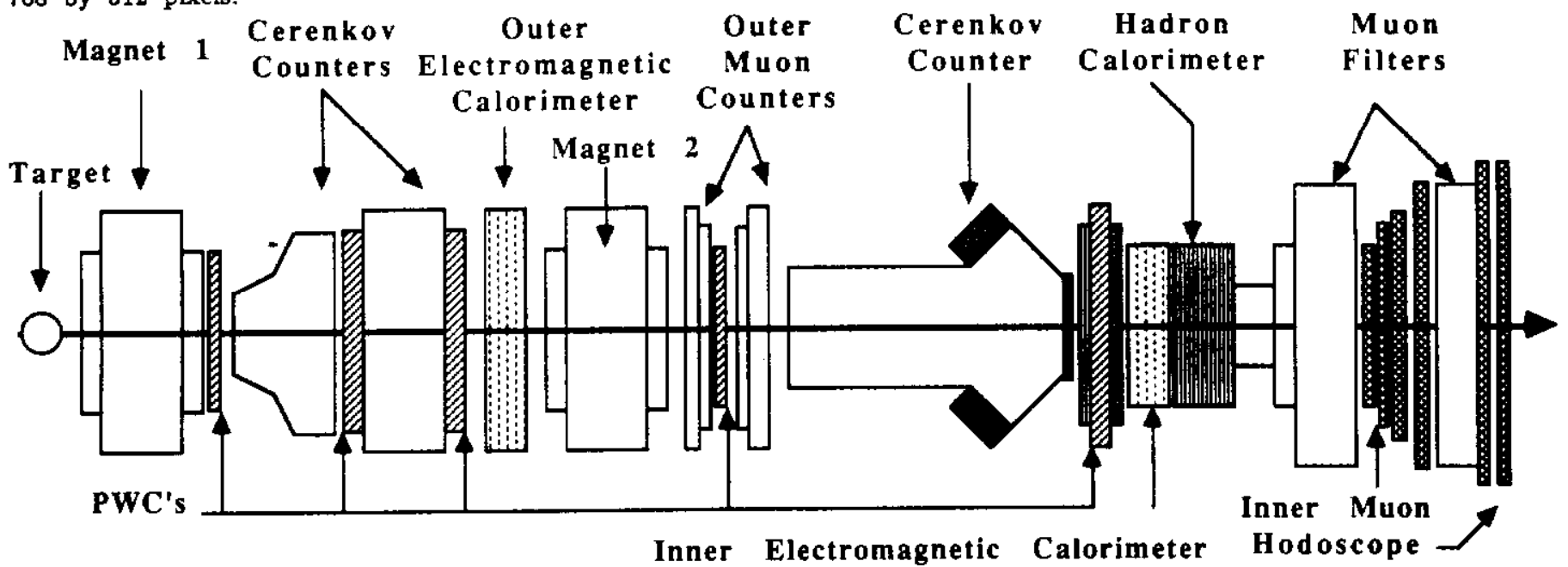

$105 \mathrm{ft}$.

Figure 1. E-687 Spectrometer configuration. The target region is shown in detail.
Target Region

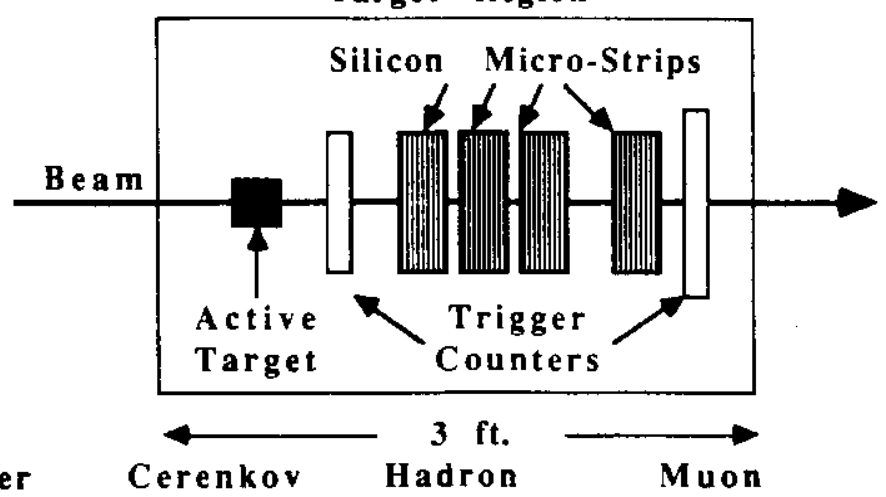

Muon t.
Video Data Acquisition System: The video imsge is converted to digital data, with 6-bit resolution, using the VDAS system developed at Fermilab. A typical interaction is shown in Figure 3 . Typical images have only 10 to 20 percent of the pixels above threshold.

In order to increase the number of images which the system can store, the data is compacted $[4,5]$ in real time and is stored locally in a large high speed FIFO. In order to be able to reconstruct the image data, VDAS atomatically inserts in the data stream horizontal and vertical sync markers as well as event tagging data. The analog-to-digital converter and data compactor have been tested at speeds of up to 30 million pixels/sec, which is as fast as our test image signal could run. It is hoped that this speed can be increased to 100 million pixels/sec in the near future. The image pixel density is programmable for any size up to 4096 by 4096 pixels.

The current FIFO is configured as 16 megabytes and has been tested at $100 \mathrm{megabytes} / \mathrm{second}$. The data is read from the FIFO by a VME based syatem and written to two streaming tape drives. The beam spill structure at Fermilab, 20 seconds of beam and 40 seconds off, dictates the FIFO size. The size of the FIFO is adjusted so that the tape drives run continuously and the FIFO buffers enough to keep the tapes busy during the 40 seconds between spills. The FIFO is a stand-alone device and can be used for buffering data from any source.

Selected events are also loaded from the VME system to an LSI-11 based image processor for display and analysis. During the running period this image processor is only used as a display device.

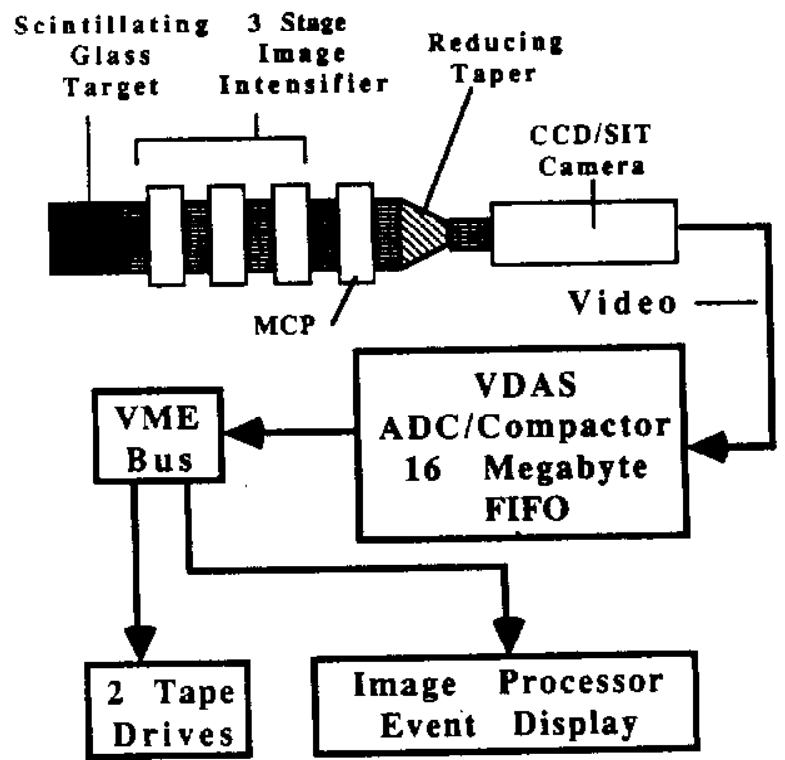

Figure 2. The Active Target, Video Data Acquisition System, and image data recording systems are shown. 


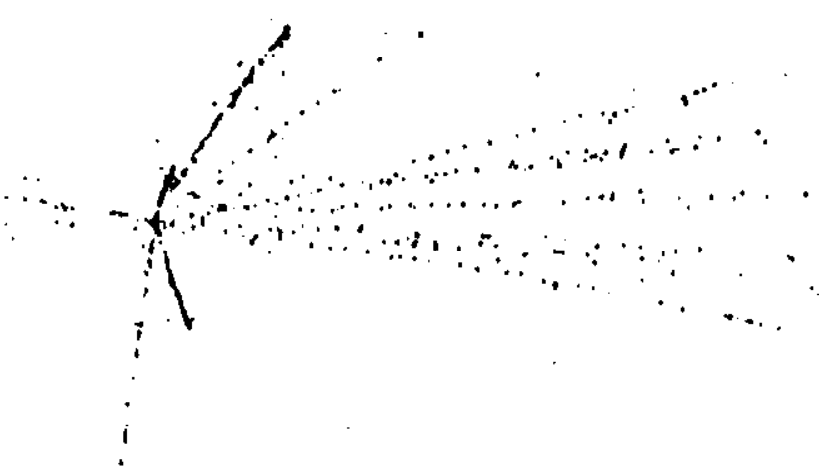

Figure 3. A Typical particle interaction image is shown. Note the low percentage of pixels which are of interest. This type of image lends itself to image compression with good results.

Data paths: The spectrometer data is read into the experimental control VAX via several separate paths. The ailicon micro-strips, calorimeters, and tracking chambers each has a data path which includes a LeCroy 1892 FIFO module.

The VDAS data path bypasses the VAX. The amount of dats involved would overwhelm the experimental data acquisition system, hence, the image data from VDAS is written to completely separate tapes.

The problem of reconstructing events is complicated by the multiple data paths, not only within the spectrometer data but also by the completely separate path for the active target image data. To solve this problem, an "Event Identification System" was designed and constructed. The system distributes a unique 64 bit ID to each data pathway which consists of 16 bit run number, a 16 bit spill number, and a 32 Bit event number within the spill. The Master ID module resides in CAMAC crate in the control system. The 64 bit ID number can be written and read via CAMAC for testing purposes. This 64 bit ID is distributed to the multiple data path via a standard backplane and adapter modules which reside in a CAMAC type crate.

In normal operation the "Run Number" is set by the VAX. Setting the run number automatically clears the spill and event number counters. The master module has inputs for TTL or NIM spill and event triggers. The "opill" input increments the spill count and clears the event counters. The "event" input simply increments the event counter. In addition to the Event ID system, VDAS has its own event tagging system. VDAS records the run, spill, and event number as part of each image frame. VDAS also records an internal frame number and byte count as part of the image data. The frame number is also exported into the spectrometer's data path and is recorded along with the data on the VAX. In this way the separate data tepes can be sorted and recombined to form complete event records.

\section{Event Analygis}

During the current running period the active target aystem will be installed for 7 shifts of beam. During this time it will record about 900,000 particle interaction images. A subset of these will be viewed in an unbiased way to observe the nature of the "average" interaction image, however, most of the events closely analyzed will be selected by examining the spectrometer data to determine interesting decay candidates. The goal is to select a few hundred interesting candidates using the spectrometer and then examine the image data for those in detail. These events will be used to fine tune the image processing algorithms so as to improve the tracking capability of the imaging aystem.
The image processing and track finding for the mass of data produced in the experiment will require an new approsch to data analysis. We are examining several possibilities to reduce the analysis time. These include large multi-processor array (the $A C P[6]$ at Fermilab) and a specialized image pipeline processor based upon a RISC chip set.

\section{VDAS Future Upgrades}

The current VDAS has several minor limitations. The system is difficult to trouble shoot in that very wide data pathways within VDAS are accomplished via ribbon cables and the number of interconnects requires that the boards have ribbon cable connectors filling both front and back edges of the boards. This requires the board set to first be assembled and then installed in a crate as a stack of boards. A single board can not be removed for testing without disassembling the entire set.

The FIFO, while electrically expandable to 256 megsbytes, in practice is limited to 16 megabytes by the cabling constraints. The other limitation of the current system is that camera configurations and setup are programmed into VDAS by a number of DIP switches within VDAS itself. Therefore, in order to change camera configurations, resolutions, speeds, etc. the system must be partially disassembled. While these limitations are not unworkable, they are an inconvenience and we are in the process of re-packaging the system.

The new syatem will reside in a Euro-card style crate $9 \mathrm{U}$ high and will have a custom backplane upon which will flow all the interconnects needed within the VDAS system. This will completely eliminate the use of ribbon cable for internal connections and will allow the individual boarda to be placed upon standard extender cards for trouble shooting and testing. A single crate will then house all of VDAS which is now in 3 separate modules. The crate will contain enough space for at least 40 megabytes of FIFO and will support additionsl communication throughout the syatem. In addition, at this time we will replace all user selectable DIP uwitches with read/writable registers so that the configuration of the oystem can be changed at will from the host computer. This will allow for the set up of several cameras and camera configuration tables so that a camera can be changed and the system reconfigured in virtually no time at all. This will make the system much more flexible and will eliminate the need to open up the system to make camera or resolution changes. The interface that will be used for this setup already exist and has till now only been used for internal testing of the VDAS hardware. This buss will now be available to the user for setup purposes.

\section{Other VDAS uses}

The VDAS system is also under consideration for other experiments, most notably a streamer chamber group at Fermilab which is proposing system that might include 36 cameras in order to get the resolution needed. The cameras would be multiplexed into 6 VDAS chassis. The system will be able to use a 6 way multiplexer since the cycle time of the streamer chamber is relatively long.

We are also investigating the possibilities for using the system for beam position measurements via synchrotron light in the SSC, and for ring imaging Cerenkov counters. Any application in which the data is in the form of an image could use VDAS, in applications currently using film the use of a VDAS would eliminate much of the problems in getting the data into a computer. The prototype VDAS system was used to study Comet Halley[7] to look for very short duration events which are masked by usual observation techniques. There is the possibility of using such a system to study astronomical events with millisecond time resolution. 
The FIFO is also of interest in any ares which has to buffer large amounts of data at high apeeds. Since it was designed to operate as atand-slone FIFO without the VDAS front-end, it can be used in many areas as a fast large buffer.

\section{Conclusions}

Although, due to scheduling at Fermilab, the current data run has not yet occurred, the active target and VDAS are in place and operational. We can, however, assess the expected performance of the system from testo done without actual beam. The total throughput for the system will be limited by the speed at which one can write data to magnetic tape, about 400,000 bytes/second. VDAS has been tested at 30 megabytes/second so the major speed limiter in the system is the mass storage device used. The compaction ratio for the images should follow that of testg using cosmic rays, about a factor of 10 , so that a typical image with 768 by 512 resolution will require about 40,000 bytes to store.

From these estimates we can assume that during the short running-period that we have, we will get nearly 1 million images recorded and that the next major task will be creating the analysis routines for handling this mass of image data.

The hardware of the current system is stable, no hardware failures have occurred in over 8 months of operation. We will be re-packaging the system in order to increase its ease of use and flexibility. This will make the maintenance of multiple VDAS systems more palatable for everyone.

While this is one application of image intensifiers and gated systems, there are many potential uses in the field of high energy physice, such as ring imaging Cerenkov counters, imaging of bubble chambers, streamer chambers, and proportional light from proportional chambers. These and other applications all require very high speed read-out systems and will become more important in the future as experiments become even more complex.

Circumatances beyond our control have prevented us from taking data with the oystem in experiment $E$ 687, we hope to be taking data very soon. All of the VDAS data paths have been verified using test images. The final test of the yystem will of course be that done with a photon beara and having the data linked with the spectrometer.

\section{References}

[1] R. Ruchti, et al., "Scintillating Glass Fiber-optic Plate Detectors for Active Target and Tracking Applications in High Energy Physics Experiments", IEEE Transactions on Nuclear Science, Vol. NS-32 No.1 (1984) pg. 590-594.

(2) R. Ruchti, et al., "a Scintillating Fiber-Optic Active Target (SFT) for Studies of High Energy Photoproduction" These proceedings.

(3) B. Baumbaugh, et al., "Control and Trigger Processors for Imaging Scintillation Detectors", These proceedings.

[4] A. Baumbaugh, et al., "A Real Time Data Compactor and 8 Megabyte High speed FIFO for HEPn. IEEE Transactions on Nuclear Science. Vol. NS-33, No. 1 (1985) pgs. 903-906

[5] K. Knickerbocker, et al., "High Speed Video Data Acquisition System (VDAS) for H.E.P..." IEEE Transactions on Nuclear Science, Vol. NS-34, No. 1 (1986) pgs. 245-249.

[6] R. Hance, et al., "The ACP Branch Bus and Real Time Applications of the ACP Multiprocessor System", IEEE Proceedinge Fifth Conference on Real Time Computer Applications in Nuclear, Particle and Plasma Physics, May 1897 (to be published), Fermilab-CONF$87 / 762380.000$ (internal memoranda).

[7] A. Baumbaugh, et al., "High Speed Video Data Acquisition System (VDAS) Developed for HEP Used to Study Comet Halley", Fermilab internal memoranda FN-441. 1986. 